\title{
Multigraphs (only) satisfy a weak triangle removal lemma
}

\author{
Asaf Shapira * Raphael Yuster ${ }^{\dagger}$ \\ Submitted: Dec 30, 2008; Accepted: Mar 5, 2009; Published: Mar 20, 2009 \\ Mathematics Subject Classification: 05C35
}

\begin{abstract}
The triangle removal lemma states that a simple graph with $o\left(n^{3}\right)$ triangles can be made triangle-free by removing $o\left(n^{2}\right)$ edges. It is natural to ask if this widely used result can be extended to multi-graphs. In this short paper we rule out the possibility of such an extension by showing that there are multi-graphs with only $n^{2+o(1)}$ triangles that are still far from being triangle-free. On the other hand, we show that for some slowly growing function $g(n)=\omega(1)$, if a multi-graph has only $g(n) n^{2}$ triangles then it must be close to being triangle-free. The proof relies on variants of the Ruzsa-Szemerédi theorem [15].
\end{abstract}

\section{Introduction}

Motivated by a problem in the theory of extremal hypergraphs, Ruzsa and Szemerédi [15] proved the following two theorems.

Theorem 1 (Ruzsa and Szemerédi [15]) If $G$ is an $n$ vertex graph from which one should remove at least $\epsilon n^{2}$ edges in order to destroy all triangles, then $G$ contains at least $f(\epsilon) n^{3}$ triangles, where $f(\epsilon)>0$.

Theorem 2 (Ruzsa and Szemerédi [15]) Suppose $S \subseteq[n]$ is a set of integers containing no 3-term arithmetic progression. Then there is a graph $G=(V, E)$ with $|V|=6 n$ and $|E|=3 n|S|$, whose edges can be (uniquely) partitioned into $n|S|$ edge disjoint triangles. Furthermore, $G$ contains no other triangles.

*School of Mathematics and College of Computing, Georgia Institute of Technology, Atlanta GA, 30332. E-mail: asafico@math.gatech.edu

${ }^{\dagger}$ Department of Mathematics, University of Haifa, Haifa 31905, Israel. E-mail: raphy@math.haifa.ac.il 
These two theorems turned out to be two of the most influential results in extremal combinatorics. First, a simple application of these two theorems gives a short proof of Roth's Theorem [14] stating that a subset of $[n]$ of size $\epsilon n$ contains a 3-term arithmetic progression. The results in [15] were followed by a long line of investigations leading to the recent hypergraph removal lemmas $[8,11,13,19]$, that also lead to new proofs of Szemerédi's Theorem [17] and some of its extensions.

Besides the above applications to additive number theory and extremal hypergraph theory, which were the original motivation for Theorems 1 and 2, they also turned out to have many additional surprising applications. In particular, these theorems also had applications in extremal combinatorics [4,7], in the study of probabilistically checkable proofs and analysis of linearity tests [9], in communication complexity [12], as well as in testing monotonicity [6] and testing graph properties [1, 2].

Theorem 1, also known as the triangle removal lemma, was originally proved for simple graphs, that is, graphs containing no parallel edges. The proof of Theorem 1 applies the regularity lemma [18], which can only handle graphs with constant edge multiplicity ${ }^{1}$. In many applications one thus has to be careful and argue that the graph (or hypergraph) on which one tries to apply Theorem 1 is indeed simple; see [16] for one such example. It is thus natural to ask if the removal lemma also holds for multi-graphs with possibly unbounded edge multiplicity. Another way of thinking about this question is whether the removal lemma holds when the edges of a graph have arbitrary weights. In multigraphs, we identify triangles by their edge sets as opposed to simple graphs where one can equivalently identify them with their vertex sets. We first show that the removal lemma does not hold in multi-graphs.

Theorem 3 There exists a multi-graph $G$ on $n$ vertices, which contains only $n^{2} 2 \sqrt{8 \log n}=$ $n^{2+o(1)}$ triangles, and yet one should remove $n^{2}$ edges from $G$ in order to make it trianglefree.

We note that the edge multiplicity of the multi-graph we use in the proof of Theorem 3 is $2 \sqrt{8 \log n}=n^{o(1)}$, so we see that the removal lemma fails even when the edge multiplicity is sub-linear in the size of the graph.

Observe that if we need to remove $n^{2}$ edges from a graph in order to make it trianglefree, then it trivially contains at least $n^{2}$ triangles. While Theorem 3 states that this trivial lower bound cannot be substantially improved, we can still ask if a minor improvement is possible. The main motivation is that in some cases (e.g., the original one in [15]) one actually only needs to know that if a graph is far from being triangle free then it contains asymptotically more than $n^{2}$ triangles. The following theorem answers this question positively. We note than in what follows we use the notation $f(n)=\omega(g(n))$ to denote the fact that $f(n) / g(n)$ goes to inifnity with $n$.

Theorem 4 If $G$ is an n-vertex multi-graph from which one should remove at least $n^{2}$ edges in order to destroy all triangles, then $G$ contains $\omega\left(n^{2}\right)$ triangles.

\footnotetext{
${ }^{1}$ The edge multiplicity of a graph is the maximum number of parallel edges between any pair of vertices.
} 
We note that because the proof of Theorem 4 applies Theorem 1 , the improvement we obtain is very minor and gives a lower bound of roughly $n^{2}\left(\log ^{*} n\right)^{c}$ for some $c>0$ on the number of triangles in the graph.

\section{The proofs}

For the proof of Theorem 3 we will need to combine Theorem 2 with the following wellknown result of Behrend [3] that was recently slightly improved by Elkin [5].

Theorem 5 (Behrend [3]) For every $n$, there exists $S \subseteq[n]$ of size $n / 2^{\sqrt{8 \log n}}=n^{1-o(1)}$ containing no 3-term arithmetic progression.

Proof of Theorem 3: Since the multigraph of Theorem 3 is derived from the graph of Theorem 2, we wish to quickly review this graph. We define a 3-partite graph $G$ on vertex sets $A, B$ and $C$, of sizes $n, 2 n$ and $3 n$ respectively, where we think of the vertices of the sets $A, B$ and $C$ as representing the sets of integers $[n],[2 n]$ and [3n]. For every $1 \leq i \leq n$ and $s \in S$ we put a triangle $T_{i, s}$ in $G$ containing the vertices $i \in A, i+s \in B$ and $i+2 s \in C$. It is easy to see that the above $n|S|$ triangles are edge disjoint, because every edge determines $i$ and $s$. To see that $G$ does not contain any more triangles, let us observe that $G$ can only contain a triangle with one vertex in each set. If the vertices of this triangle are $a \in A, b \in B$ and $c \in C$, then we must have $b=a+s_{1}$ for some $s_{1} \in S$, $c=b+s_{2}=a+s_{1}+s_{2}$ for some $s_{2} \in S$, and $a=c-2 s_{3}=a+s_{1}+s_{2}-2 s_{3}$ for some $s_{3}$. This means that $s_{1}, s_{2}, s_{3} \in S$ form an arithmetic progression, but because $S$ is free of 3 -term arithmetic progressions it must be the case that $s_{1}=s_{2}=s_{3}$ implying that this triangle is one of the triangles $T_{i, s}$ defined above.

Let, therefore, $G^{\prime}$ be the graph of Theorem 2 when taking $S \subseteq[n]$ to be a $3 A P$-free set of size $n / 2^{\sqrt{8 \log n}}$ as guaranteed by Theorem 5 . Let $G$ be the graph obtained by replacing every edge of $G^{\prime}$ with $n /|S|=2^{\sqrt{8 \log n}}$ parallel edges. Observe that as $G^{\prime}$ contains $n|S|$ edge disjoint triangles, one must remove at least $n|S|$ edges from it in order to make it triangle-free. As $G$ contains $n /|S|$ parallel edges for every edge of $G^{\prime}$ we infer that one must remove $n^{2}$ edges from $G$ in order to make it triangle-free. Finally, as $G^{\prime}$ contains only $n|S|$ triangles, we infer that $G$ contains only $n|S|(n /|S|)^{3}=n^{2} 2^{2 \sqrt{8 \log n}}$ triangles, as needed.

Proof of Theorem 4: Given a multi-graph $G$, let $T$ be the simple graph on the same vertex set that contains an edge $(u, v)$ if and only if $G$ has at least one but at most $g^{2}(n)$ edges connecting $u$ and $v$ for some function $g(n)=\omega(1)$ to be chosen shortly. Let's first consider the case that one needs to remove at least $\frac{1}{2 g^{2}(n)} n^{2}$ edges from $T$ in order to make it triangle-free. In this case, by Theorem 1, we know that $T$ contains at least $f\left(\frac{1}{2 g^{2}(n)}\right) n^{3}$ triangles. Let us now choose a function $g(n)=\omega(1)$ such that $f\left(\frac{1}{2 g^{2}(n)}\right) n^{3}=\omega\left(n^{2}\right)$. This is clearly possible no matter how fast $f(\epsilon)$ goes to 0 with $\epsilon$. Specifically, given the known 
bounds on $f(\epsilon)$ in Theorem 1 (see, e.g., [10]), one can take $g(n)=\left(\log ^{*} n\right)^{c}$ for some constant $c>0$. Fixing this choice of $g(n)$ guarantees that in this case $T$ contains $\omega\left(n^{2}\right)$ triangles and so $G$ contains at least this many triangles as well.

So we can assume that we can remove from $T$ a set of edges $E$ of size $\frac{1}{2 g^{2}(n)} n^{2}$ and thus make it triangle-free. Let us now remove from $G$ all the edges connecting pairs of vertices that are connected by $E$ in $T$. Note that we thus remove from $G$ at most $g^{2}(n) \cdot \frac{1}{2 g^{2}(n)} n^{2} \leq n^{2} / 2$ edges, hence, by the hypothesis of the theorem, the new graph we obtain, let's call it $G^{\prime}$, has the property that we should remove at least $n^{2} / 2$ edges from it in order to make it triangle-free. Furthermore, each edge in $G^{\prime}$ has multiplicity at least $g^{2}(n)$.

Let $T^{\prime}$ be the simple graph underlying $G^{\prime}$, that is, the graph on the same vertex set, with an edge $(u, v)$ if and only if $G^{\prime}$ has an edge between $u$ and $v$. Assume first that $T^{\prime}$ contains at least $n^{2} / g(n)$ edges that belong to a triangle. In this case $T^{\prime}$ contains at least $n^{2} / 3 g(n)$ triangles, and as the edge multiplicity of $G^{\prime}$ is at least $g^{2}(n)$ this means that $G^{\prime}$ contains at least $n^{2} g(n) / 3$ triangles. As $G^{\prime}$ is a subgraph of $G$ we infer that $G$ also contains $n^{2} g(n) / 3$ triangles.

So we can now assume that $T^{\prime}$ has at most $n^{2} / g(n)$ edges that belong to a triangle. Let $E^{\prime}$ be a set of minimal size whose removal from $G^{\prime}$ makes it triangle-free. Let $B$ denote the set of pairs $(u, v)$ for which $E^{\prime}$ contains at least one edge connecting $u$ and $v$, and note that by our assumption on $T^{\prime}$ we have that $|B| \leq n^{2} / g(n)$. For each pair of vertices $(u, v) \in B$ let $m_{u, v}$ be the number of edges connecting $u$ and $v$ that belong to $E^{\prime}$. We claim that for every $(u, v)$ there are at least $m_{u, v}$ paths of length exactly 2 connecting $u$ and $v$. Indeed, if $G^{\prime}$ contains less than $m_{u, v}$ such paths, then we can remove the $m_{u, v}$ edges connecting $u$ and $v$ from $E^{\prime}$ and replace them by one edge from each of the paths of length 2 connecting $u$ and $v$. The new set has fewer edges and it still makes $G^{\prime}$ triangle-free, which contradicts the minimality of $E^{\prime}$. We thus conclude that for every pair $u, v$ the graph $G^{\prime}$ has at least $m_{u, v}^{2}$ triangles containing $u$ and $v$. Recall that $G^{\prime}$ still has the property that one should remove at least $n^{2} / 2$ edges from it in order to make it triangle-free. Therefore we have $\sum m_{u, v}=\left|E^{\prime}\right| \geq n^{2} / 2$. Combining the above facts, and using Cauchy-Schwartz, we conclude that the number of triangles in $G^{\prime}$ (and so also in $G)$ is at least

$$
\sum_{(u, v) \in B} m_{u, v}^{2} \geq \frac{1}{|B|}\left(\sum_{(u, v) \in B} m_{u, v}\right)^{2} \geq \frac{1}{4|B|} n^{4} \geq \frac{1}{4} g(n) n^{2},
$$

thus completing the proof.

\section{References}

[1] N. Alon, Testing subgraphs in large graphs, Random structures and algorithms, 21 (2002), 359-370.

[2] N. Alon and A. Shapira, A characterization of easily testable induced subgraphs, Combinatorics, Probability and Computing, 15 (2006), 791-805. 
[3] F. A. Behrend, On sets of integers which contain no three terms in arithmetic progression, Proc. National Academy of Sciences USA 32 (1946), 331-332.

[4] L. H. Clark, R. C. Entringer, J. E. McCanna and L. Székely, Extremal problems for local properties of graphs, Combinatorial Mathematics and Combinatorial Computing (Palmerston North, 1990). Australas. J. Combin. 4 (1991), 25-31.

[5] M. Elkin, An improved construction of progression-free sets, arxiv.org/abs/0801.4310 .

[6] E. Fischer, E. Lehman, I. Newman, S. Raskhodnikova, R. Rubinfeld and A. Samorodnitsky, Monotonicity testing over general poset domains, Proc. of STOC 2002, 474483.

[7] Z. Füredi, The maximum number of edges in a minimal graph of diameter 2, J. of Graph Theory 16 (1992), 81-98.

[8] T. Gowers, Hypergraph regularity and the multidimensional Szemerédi theorem, Ann. of Math. Volume 166, Number 3 (2007), 897-946.

[9] J. Håstad and A. Wigderson, Simple analysis of graph tests for linearity and PCP, Random Structures and Algorithms, 22 (2003), 139-160.

[10] J. Komlós and M. Simonovits, Szemerédi's Regularity Lemma and its applications in graph theory. In: Combinatorics, Paul Erdős is Eighty, Vol II (D. Miklós, V. T. Sós, T. Szönyi eds.), János Bolyai Math. Soc., Budapest (1996), 295-352.

[11] B. Nagle, V. Rödl and M. Schacht, The counting lemma for regular $k$-uniform hypergraphs, Random Structures and Algorithms 28 (2006), 113-179.

[12] P. Pudlák and J. Sgall, An upper bound for a communication game related to timespace tradeoffs, The mathematics of Paul Erdős, I, (R. L. Graham and J. Nešetřil, eds.), Algorithms Combin., 13, Springer, Berlin, (1997), 393-399.

[13] V. Rödl and J. Skokan, Regularity lemma for $k$-uniform hypergraphs, Random Structures and Algorithms 25 (2004), 1-42.

[14] K.F. Roth, On certain sets of integers, J. London Math. Soc. 28 (1953), 104-109.

[15] I. Ruzsa and E. Szemerédi, Triple systems with no six points carrying three triangles, in Combinatorics (Keszthely, 1976), Coll. Math. Soc. J. Bolyai 18, Volume II, 939945.

[16] A. Shapira, A proof of Green's conjecture regarding the removal properties of sets of linear equations, submitted.

[17] E. Szemerédi, Integer sets containing no $k$ elements in arithmetic progression, Acta Arith. 27 (1975), 299-345.

[18] E. Szemerédi, Regular partitions of graphs, In: Proc. Colloque Inter. CNRS (J. C. Bermond, J. C. Fournier, M. Las Vergnas and D. Sotteau, eds.), 1978, 399-401.

[19] T. Tao, A variant of the hypergraph removal lemma, J. Combin. Theory, Ser. A 113 (2006), 1257-1280. 